\title{
Congratulations to the 2018 Recipients of Foundation Grants and Scholarships
}

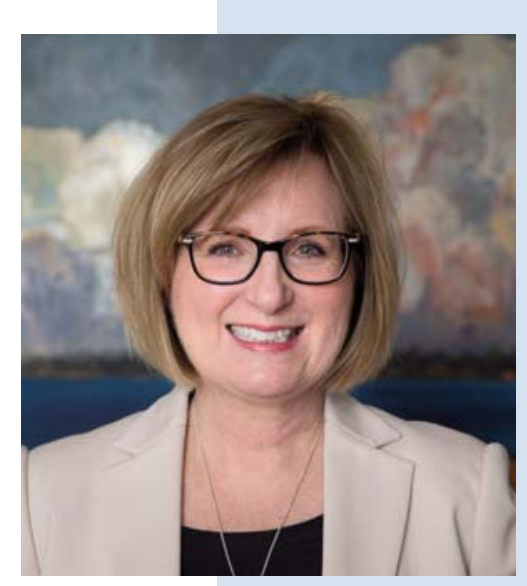

\section{Theresa Schindel}

Associate Clinical Professor, Faculty of Pharmacy and

Pharmaceutical Sciences, University of Alberta, Edmonton,

Alberta

Exploring the Role and Experiences of Hospital Pharmacists

Who Care for Patients Who Seek Medical Assistance in Dying

Research Grant: \$14,530

Facility: University of Alberta

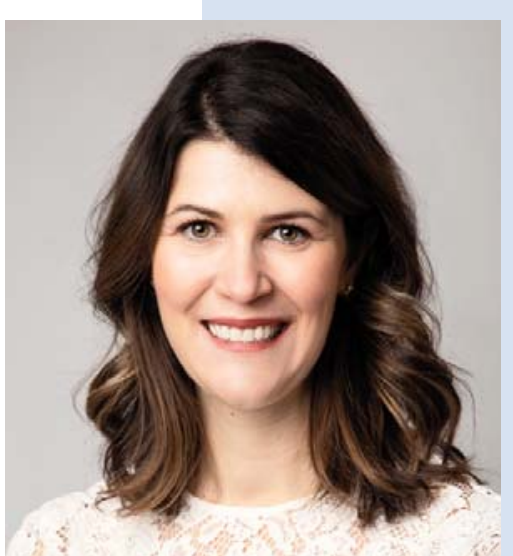

\section{Laura Murphy}

Pharmacy Clinical Leader, Toronto Rehabilitation Institute,

University Health Network, Toronto, Ontario

Examining the Relationship amongst Opioid Subjective Effects and Pharmacokinetics of Extended Release Opioids at Shortened Dosing Intervals in Patients with Chronic Pain: A Randomized, Blinded, n-of-1 Case Series Feasibility Study

Research Grant: \$8,844

Facility: Toronto Rehabilitation Institute, University Health Network

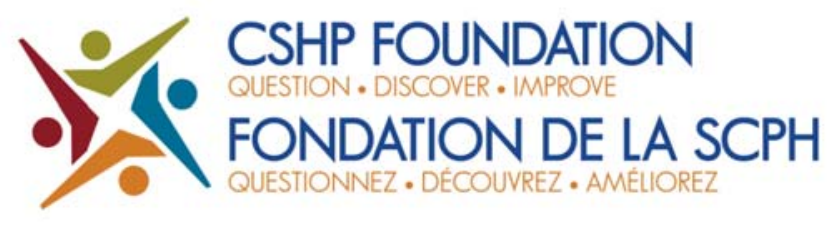




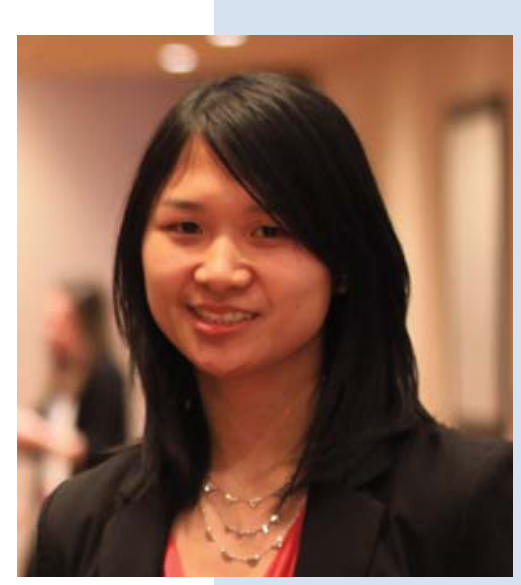

\section{Vivian Law}

Clinical Pharmacist, Orthopaedics/Neurology and Multiple Sclerosis Clinic, St. Michael's Hospital, Toronto, Ontario

Traineeship: Advanced training in multiple sclerosis treatment

Education Grant: $\$ 6,400$

Facility: Multiple Sclerosis Clinic, University of Colorado Hospital

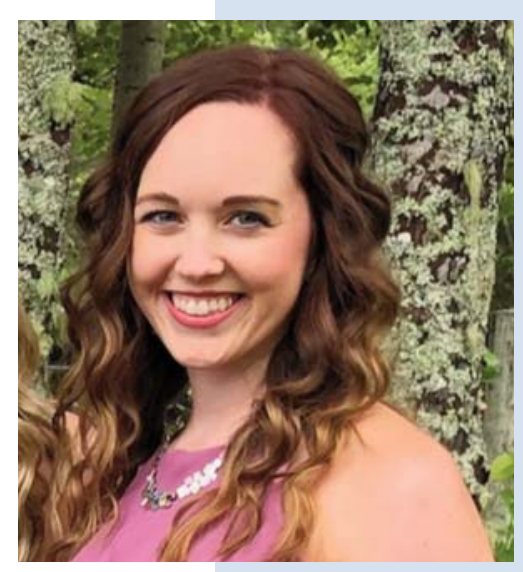

\section{Megan Riordon}

Pharmacy Manager, Clinical Practice, Kingston Health Sciences Centre, Kingston, Ontario

Scholarship: ASHP Pharmacy Leadership Academy

The Pharmacy Leadership Academy courses offer a range of critical topics, incorporating self-learning and reading, presentations, and case-based interactive components to inform and stimulate mentees with the objective of improving patient care through the enhancement of leadership skills, which can be applied at every level of a healthcare organization.

Education Scholarship: US \$6,650

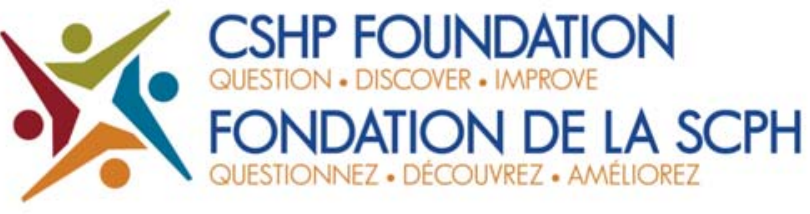

If every CSHP member were to donate $\$ 25$ in 2019, the Foundation would have $\$ 70,000$ to fund future grants and scholarships.

\section{Donate to the Foundation now at: cshpfoundation.ca}

